OPEN ACCESS

Edited by:

Ayako N. Sakamoto,

National Institutes for Quantum and Radiological Science and Technology, Japan

Reviewed by:

Jiangwei Yang,

Gansu Agricultural University, China

Fei Gao,

Minzu University of China, China

Hongxia Zhang,

Ludong University, China

*Correspondence:

Xiaoxu L

82101171073@caas.cn

Yongfeng Guo

guoyongfeng@caas.cn

${ }^{\dagger}$ These authors have contributed

equally to this work

Specialty section:

This article was submitted to

Plant Abiotic Stress,

a section of the journal

Frontiers in Plant Science

Received: 03 December 2020

Accepted: 19 April 2021

Published: 28 May 2021

Citation:

Wang Q, Guo C, Li Z, Sun J,

Wang D, XU L, Li X and Guo Y (2021)

Identification and Analysis of bZIP

Family Genes in Potato and Their

Potential Roles in Stress Responses.

Front. Plant Sci. 12:637343.

doi: 10.3389/fpls.2021.637343

\section{Identification and Analysis of bZIP Family Genes in Potato and Their Potential Roles in Stress Responses}

\author{
Qi Wang1,2†, Cun Guo ${ }^{1,2+}$, Zhiyuan Li1,2, Jinhao Sun 1,2, Dong Wang ${ }^{3}$, Liangtao X ${ }^{3}$, \\ Xiaoxu $\mathrm{Li}^{1,3 *}$ and Yongfeng Guo ${ }^{1 *}$ \\ ${ }^{1}$ Tobacco Research Institute, Chinese Academy of Agricultural Sciences, Qingdao, China, ${ }^{2}$ Graduate School of Chinese \\ Academy of Agricultural Sciences, Beijing, China, ${ }^{3}$ Technology Center, China Tobacco Hunan Industrial Co., Ltd., \\ Changsha, China
}

The bZIP proteins comprise one of the largest transcription factor families and play important roles in plant growth and development, senescence, metabolic reactions, and stress responses. In this study, 49 bZIP transcription factor-encoding genes (StbZIP genes) on the potato genome were identified and analyzed. The 49 StbZIP genes, which are located on 12 chromosomes of the potato genome, were divided into 11 subgroups together with their Arabidopsis homologs based on the results of phylogenetic analysis. Gene structure and protein motif analysis revealed that members from the same subgroup often possessed similar exon/intron structures and motif organizations, further supporting the results of the phylogenetic analysis. Syntenic analysis indicated the existence of gene duplication events, which might play an important role in the expansion of the bZIP gene family in potato. Expressions of the StbZIP genes were analyzed in a variety of tissues via RNA-Seq data, suggesting functional diversity. Several StbZIP genes were found to be induced by different stress conditions. For example, the expression of StbZIP25, the close homolog of AtbZIP36/ABF2, was significantly upregulated by salt stress treatments. The StbZIP25 protein was found to be located in the nucleus and function as a transcriptional activator. Overexpression of StbZIP25 enhanced salt tolerance in Arabidopsis. The results from this study imply potential roles of the bZIP family genes in the stress response of potato.

Keywords: potato, bZIP, abiotic stress, transcription factor, gene family

\section{INTRODUCTION}

In the plant kingdom, transcription factors regulate various biological processes by activating or repressing gene expression (Qu and Zhu, 2006), among which the basic leucine zipper (bZIP) transcription factors have been reported to be one of the most diverse transcription factor families in plants, regulating various developmental processes and stress responses (Wang et al., 2015; Droge-Laser et al., 2018). The bZIP transcription factors possess a conserved bZIP domain, which is composed of 60-80 amino acids, including a DNA-binding basic region and a leucine (Leu) zipper domain (Landschulz et al., 1988; Droge-Laser et al., 2018). The basic region is highly conserved and consists of approximately 16 amino acid residues, containing an invariant N-X7-R/K-X9 motif that binds to specific DNA sequences with an ACGT core, such as A-box (TACGTA), C-box 
(GACGTC), and G-box (CACGTG) (Izawa et al., 1993; Niu et al., 1999). The Leu zipper domain is less conserved and consists of heptad repeats of leucine or other hydrophobic amino acids, which play an important role in specific recognition and dimerization (Jakoby et al., 2002; Wei et al., 2012; Wang et al., 2015).

In the model plant Arabidopsis, the bZIP family members have been classified into 13 groups, and most of them have been verified to have specific functions in regulating plant development (Droge-Laser et al., 2018). In group A, AtbZIP12/EEL and AtbZIP39/ABI5 regulate gene expression through competing for the same binding sites within the promoter of the AtEm1 gene during late embryogenesis (Bensmihen et al., 2002). AtbZIP14/FD can form a complex with FLOWERING LOCUS T (FT) in controlling flowering by activating the floral identity gene APETALA1 (AP1) (Wigge et al., 2005). In group C, AtbZIP9 was reported to be involved in vascular development in roots (Silveira et al., 2007). In group I, AtbZIP18 is a pollen-expressed bZIP transcription factor exhibiting functional redundancy with AtbZIP34 in male gametophyte development (Gibalova et al., 2017). AtbZIP29 was reported to function in leaf and root development (van Leene et al., 2016). AtbZIP30/DKM from group I negatively regulates Arabidopsis growth and reproductive development (Lozano Sotomayor et al., 2016). The size of $\mathrm{dkm}$ mutants was larger than that of wild-type individuals, with more floral buds, while compared to wild-type plants, $D K M$ overexpression plants displayed a reduction in floral bud number (Lozano Sotomayor et al., 2016). AtbZIP44 in group S was reported to participate in regulating seed germination (Iglesias Fernandez et al., 2013).

Leaf senescence is a programmed developmental process and largely affects the yield and nutritional value of food crops (Gan and Amasino, 1997; Guo and Gan, 2014). The detached leaves of the triple mutant (abf2abf3abf4) displayed a staygreen phenotype after abscisic acid (ABA) treatment, indicating that AtbZIP36/ABF2, AtbZIP37/ABF3, and AtbZIP38/ABF4 in group A positively regulate ABA-mediated leaf senescence in Arabidopsis (Gao et al., 2016). AtbZIP41/GBF1 in group G was highly expressed during leaf senescence and was reported to regulate the onset of leaf senescence via reducing the expression of the CAT2 gene (Smykowski et al., 2010). Besides, AtbZIP1 and AtbZIP53 in group Scan synergistically regulate dark-induced leaf senescence (Dietrich et al., 2011). bZIP members have also been reported to be involved in plant metabolism. For example, in Arabidopsis, AtbZIP56/HY5 from group $\mathrm{H}$ was reported to regulate the biosynthesis of anthocyanins by binding the promoters of some $M Y B$ genes or inducing the expressions of biosynthesis enzyme genes (Shin et al., 2013). Similarly, in tomato, the bZIP transcription factor HY5 was also found to be involved in CRY1a-induced anthocyanin biosynthesis (Liu et al., 2018).

Furthermore, several bZIP proteins were reported to function in response to abiotic/biotic stresses in Arabidopsis (Banerjee and Roychoudhury, 2017). In group A, AtbZIP35/ABF1 was highly upregulated by cold stress (Roychoudhury and Paul, 2012). AtbZIP36/ABF2 was reported to be involved in abiotic stress responses by regulating the expressions of stress-regulated genes
(Kim et al., 2010). AtbZIP17 in group B and AtbZIP24 in group F were also reported to enhance salt tolerance via regulating stress response genes (Liu et al., 2008; Yang et al., 2009). AtbZIP51/VIP1 from group I was found to regulate osmotic pressure by directly binding to the promoters of CYP707A1/3 genes (Tsugama et al., 2012). In group $S$, the expression of $A t b Z I P 1$ was significantly upregulated under salt, cold, and drought stresses, and transgenic Arabidopsis plants overexpressing AtbZIP1 had an enhanced tolerance to salt and drought stresses, indicating that AtbZIP1 functions as a positive regulator in plants in response to abiotic stresses (Sun et al., 2012). AtbZIP62, on the other hand, was reported to serve as a negative regulator of salt stress (Rolly et al., 2020).

Potato is an important economic crop all over the world. Increasing the resistance of potato to biotic/abiotic stresses for yield increase has been a hot research topic. With the availability of the Solanaceae genome database, studies of a number of potato gene families have been reported (Muniz Garcia et al., 2012; Zhou et al., 2018), but our understanding of the potato $b Z I P$ gene family is very limited. In this study, we have identified 49 potato bZIP genes from the Solanaceae database. Comprehensive analyses on the gene structure, promoter, chromosome distribution, phylogenetic analysis, and expression patterns were conducted, the results of which suggested that the potato bZIP members may play various roles in potato development and in response to stresses.

\section{MATERIALS AND METHODS}

\section{Identification and Classification of bZIP Genes in Potato}

The potato genome annotations (PGSC, release 3.4) were retrieved from the Sol Genomics Network $(\mathrm{SGN})^{1}$. Two different methods were used to identify the bZIP genes in potato: (1) BLASTP search using the Arabidopsis bZIP protein sequences according to previous methods (Li et al., 2018) and (2) the hidden Markov model profiles of bZIP domains (PF00170, PF07716, and PF03131) downloaded from Pfam (El Gebali et al., 2019) and used in searching potato proteome sequences via the HMMER (Johnson et al., 2010) program with an $E$-value cutoff of 0.001 . All outputted bZIP protein sequences from the two methods were confirmed to have the bZIP domains using both InterProScan (Quevillon et al., 2005) and SMART (Letunic and Bork, 2018) databases. Candidate sequences that do not contain bZIP domains were manually deleted, and the remaining sequences were named according to their chromosome locations. Subsequently, potato bZIP protein sequences were submitted to the online tool ProtParam (Wilkins et al., 1999) to predict amino acid quantity, molecular weight, and isoelectric point.

Phylogenetic analysis was performed using the previously reported Arabidopsis bZIP sequences (Droge-Laser et al., 2018) and the newly identified potato bZIP protein sequences. Multi-sequence alignment was performed using MAFFT

${ }^{1}$ http://solgenomics.net/ 
(Katoh et al., 2019), and the result was presented with the Texshade program (Beitz, 2000). Based on the alignment results, MEGA was used to build a neighbor-joining (NJ) tree under the default parameters. The FigTree software was employed to visualize the tree file (University of Maryland, College Park, MD, United States).

\section{Exon-Intron Structural Analysis and Identification of Conserved Motifs}

The Gene Structure Display Server (Hu et al., 2015) was used to visualize the exon-intron structures of the Arabidopsis and potato bZIP genes by submitting their genomic and coding sequences. The conserved motifs of their protein sequences were identified via Multiple Em for Motif Elicitation (MEME) tools (Bailey et al., 2009) with the following parameters: distribution of motif occurrences, zero or one per sequence; maximum number of motifs, 10; and the optimum width of each motif, between six and 100 residues.

\section{Chromosomal Localization and Duplication Event Analysis of Potato bZIP Genes}

The chromosomal location information of potato bZIP genes were downloaded from the SGN database and displayed visually with Perl. Tandem gene events were defined as previously described (Li et al., 2018) and displayed on the potato chromosomes. For syntenic analysis, we searched the synteny relationship of the orthologous genes from potato and four other plant species (including Arabidopsis, tomato, grape, and rice), and the results were displayed with TBtools (Chen et al., 2020). Subsequently, the synonymous substitution (Ks) and nonsynonymous substitution $(\mathrm{Ka})$ rates were calculated using the DnaSP 5.0 software (Librado and Rozas, 2009).

\section{Analysis of cis-Elements in the Promoter of Potato bZIP Genes}

To assess the promoter cis-acting elements of the StbZIP genes, $2,000 \mathrm{bp}$ of promoter regions upstream of the start codon of the StbZIP genes were extracted. PlantCARE ${ }^{2}$ was engaged for cisacting regulatory element investigation.

\section{Expression Pattern Analysis of Potato bZIP Genes}

To determine the expressions of StbZIP genes in different tissues, RNA sequencing (RNA-Seq) data were obtained from the PGSC database (Xu et al., 2011). The expression data of the root, stem, shoot apex, leaf, flower, and tuber were selected. The expression data of biotic and abiotic stresses were also downloaded and the relative expression calculated relative to their controls, respectively. Only genes with absolute expression values of log multiples $>2$ were displayed. The resulted data were normalized and illustrated by $\mathrm{R}$. This $\mathrm{R}$ package has been integrated in TBtools (Chen et al., 2020).

\footnotetext{
${ }^{2}$ http://bioinformatics.psb.ugent.be/webtools/plantcare/html/
}

\section{Potato Plant Preparation and Salt Stress Treatments}

The potato cultivar GN2 (Gannongshu 2) was used to analyze the expressions of StbZIP genes. Potato sprouts were incubated on complete Murashige and Skoog (MS) solid medium by nodule cutting and cultivated in a growth chamber at $24^{\circ} \mathrm{C}$ under continuous light $\left(70 \pm 5 \mu \mathrm{mol} \mathrm{m}{ }^{-2} \mathrm{~s}^{-1}\right.$ photosynthetic photon flux density) and $50-60 \%$ relative humidity. For salt stress treatments, 4-week-old seedlings were treated with $150 \mathrm{mM}$ $\mathrm{NaCl}$. After treatments of $0,1,3$, and $6 \mathrm{~h}$, whole plants were harvested for RNA extraction and real-time PCR (RT-PCR) analysis. The harvested samples were immediately frozen in liquid nitrogen and stored at $-80^{\circ} \mathrm{C}$ prior to RNA extraction. Three biological replicates were used for each sample.

\section{RNA Extraction and Real-Time Polymerase Chain Reaction Analysis}

Total RNAs were extracted using the Ultrapure RNA Kit (cwbiotech, Beijing, China), and the first-strand complementary DNA (cDNA) was synthesized using the PrimeScript ${ }^{\mathrm{TM}}$ RT reagent Kit (TaKaRa). The potato EFl $\alpha$ gene was adopted as an internal control (Singh et al., 2013), and quantitative RT-PCR (qRT-PCR) reactions were performed with 40 cycles in a Roche LightCycler 480 Real-Time PCR instrument. All expression data were obtained from three technical repeats and calculated by the $2^{-\Delta \Delta C T}$ method. Statistical significance was analyzed using SPSS v18.0 $t$ test. The primer sequences used in the current study are listed in Supplementary Table 1.

\section{Subcellular Localization and Transactivation Assays}

The StbZIP25 coding sequence (CDS) without stop codon was PCR amplified from potato cDNAs and cloned into the pEasy-Blunt subcloning vector then inserted into the PYG57 vector, which contains the green fluorescent protein (GFP) coding sequence, to construct the StbZIP25-GFP fusion gene driven by the CaMV-35S promoter. The construct was then transformed into Agrobacterium GV3101 competent cells and transiently expressed in the leaves of Nicotiana benthamiana. Simultaneously, the empty PYG57 vector was used as a control. Three days after injection, the leaves were soaked in the DAPI staining solution and observed under a confocal laser microscope (TCS-SP8 Leica, Wetzlar, Germany) to detect fluorescence signals of the fusion protein, as previously reported (Li et al., 2019a,b).

For the transactivation activity assay of StbZIP25, the fulllength CDS of the StbZIP25 gene was PCR amplified and inserted into the pBridge vector at the EcoRI site by Infusion (Clontech, Bejing, China) to fuse with a GAL4 DNA binding domain, yielding plasmid pBridge-StbZIP25. Plasmid pBridge-StbZIP25 and the empty pBridge vector (control) were then transformed into the yeast strain AH109, and the transformed yeasts were plated on synthetic dextrose (SD) media lacking tryptophan (SD/-Trp) and incubated at $30^{\circ} \mathrm{C}$ for 3 days. Subsequently, the positive clones were transferred to $\mathrm{SD} /$-Trp media supplemented with $\mathrm{X}$-gal and incubated at $30^{\circ} \mathrm{C}$ for 3 days. The transactivation 
activity of the fused proteins was determined based on the growth status (blue/white) of the transformants.

\section{Overexpression Analysis}

The StbZIP25 gene coding sequence was PCR amplified and inserted into the pCHF3 vector to generate the StbZIP25 overexpression construct driven by the CaMV-35S promoter, which was then transformed into Arabidopsis wild-type (Col0) plants by the floral dip method (Zhang et al., 2006). The T0 generation seeds were screened on half-strength MS medium with $50 \mathrm{mg} / \mathrm{L}$ kanamycin to obtain StbZIP25 overexpression plants. Seven-day-old T3 transgenic and wildtype (WT) Arabidopsis plants were transferred to 1/2 MS media with or without $\mathrm{NaCl}(100 \mathrm{mM})$ to be grown vertically in a growth chamber $\left(23^{\circ} \mathrm{C}\right.$, continuous light). The primary root length of 18 plants was measured after 21 days. Significant difference analysis was calculated by SPSS v18.0 with the $t$ test program.

\section{RESULTS}

\section{Identification of StbZIP Genes}

The BLASTp method was used to identify $b Z I P$ genes from the potato genome. A total of 49 StbZIP genes were identified after determining the presence of a complete bZIP motif in each candidate. The 49 StbZIP genes were distributed on 12 potato chromosomes and were named from StbZIP01 to StbZIP49, according to their chromosomal locations.

Characteristics of the StbZIP proteins including the number of amino acids, molecular weight $\left(M_{w}\right)$, and the isoelectric point ( $\mathrm{p} I$ ) were analyzed (Supplementary Table 2). The length of the 49 StbZIP proteins ranged from 132 (StbZIP12) to 822 (StbZIP46) amino acids. The molecular weight of the bZIP proteins ranged from 15.41 (StbZIP04 and StbZIP05) to 88.18 (StbZIP46) kDa, and $\mathrm{p} I$ ranged from 5.02 (StbZIP35) to 10.31 (StbZIP07).

\section{Multiple Sequence Alignment and Phylogenetic Analysis}

In plants, the bZIP proteins possess a highly conserved DNAbinding basic region. To investigate the characteristics of the potato bZIP members, full-length sequences of all the newly identified StbZIP proteins were subjected to multiple sequence alignments, and the basic region was visualized by $\mathrm{R}$. The basic region possesses a nuclear localization signal and an invariant $\mathrm{N}-\mathrm{X} 7-\mathrm{R} / \mathrm{K}-\mathrm{X} 9$ motif (Supplementary Figure 1A). In addition, the characteristics of the basic region sequence were highly conserved between potato and Arabidopsis (Supplementary Figures 1A,B).

To explore the phylogenetic relationship of the StbZIP proteins, a neighbor-joining tree was constructed based on multiple sequence alignment of the 49 potato bZIP members and their Arabidopsis homologs (Figure 1). Based on the results of the phylogenetic analysis and the results of a previous study in which Arabidopsis bZIP members were classified into 13 groups (Droge-Laser et al., 2018), the 49 potato bZIP proteins were classified into 11 groups together with their Arabidopsis homologs. Notably, most of the groups contained two or more potato bZIP members, indicating that the differentiation time of potato and Arabidopsis was later than that of the bZIP gene family. Among the groups, group $S$ contained the largest number of members (15), while groups $\mathrm{C}$ and $\mathrm{H}$ contained the minimum number of members (one). Intriguingly, it was found that only the Arabidopsis bZIP proteins fall into groups D and J, indicating that the StbZIP genes in these groups might have been lost during the evolution of potato.

\section{StbZIP Genes Syntenic Analysis}

To further study the evolutionary relationship among the potato bZIP genes, syntenic analysis was carried out for potato and four other plant species, including three dicots (Arabidopsis, tomato, and grape) and one monocot (rice) (Figure 2A). The results showed that there was a syntenic relationship between 45 of the StbZIP genes with the bZIP genes in tomato, 38 StbZIP genes in grape, 26 StbZIP genes in Arabidopsis, and eight StbZIP genes in rice. The numbers of predicted collinear pairs between potato and tomato, grape, Arabidopsis, and rice, were 81, 56, 44, and 14, respectively.

Seven StbZIP genes were predicted to form collinear pairs with the $b Z I P$ genes of all the other four species, which indicates that these genes may have existed before the differentiation of these species and have maintained a collinear relationship since then. It is worth noting that a total of 18 collinear gene pairs were identified between potato and tomato/grape/Arabidopsis species, but no collinear gene pair was found in the rice genome, indicating that those pairs may have appeared after the divergence of dicots and monocots. Interestingly, 12 AtbZIP genes were predicted to have a collinearity relationship with two or more StbZIP genes, which implies that the formation of these StbZIP genes may be caused by duplications and might have played significant roles in the evolution of potato (Figure $\mathbf{2 B}$ and Supplementary Table 3).

\section{Gene Structure and Motif Composition}

As shown in Supplementary Figure 2, the gene structures of potato and the Arabidopsis bZIP genes were drawn with the online software GSDS and displayed visually. The numbers of introns of these genes range from zero to 11. Among them, 15 potato bZIP genes have no introns. Interestingly, all the genes without intron were classified into group S. In addition, the genes with the largest number of introns were distributed in group $\mathrm{G}$, and the average number of introns in this group is 10 . The genes of groups A and I all contain two to three introns, while group $\mathrm{C}$ genes contain five to six introns. bZIP genes of the same group have similar gene structures, indicating that the abovedescribed evolutionary relationship and classification analysis of StbZIP genes were reliable. It is worth mentioning that, although the average number of introns in Arabidopsis (10.6) and potato (10.5) is similar, group D, which only contains Arabidopsis genes, shows the largest variation in gene structure, with the number of introns ranging from 6 to 11 .

Subsequently, the MEME online tool was used to analyze the conserved domains of potato and Arabidopsis bZIP proteins. A total of 10 conserved functional domains were identified 


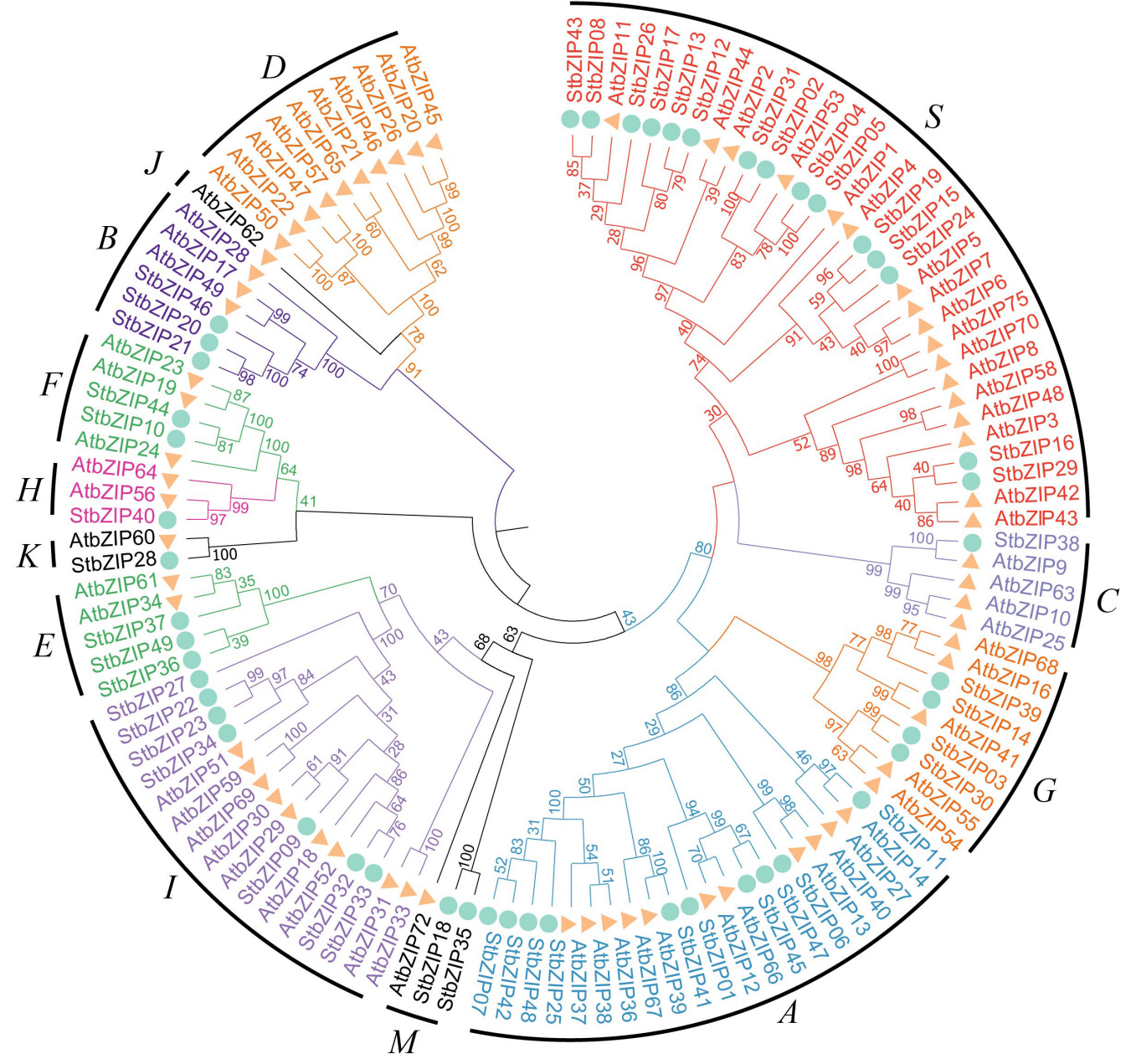

FIGURE 1 | Phylogenetic analysis of potato bZIP family members. The phylogenetic tree was generated from the alignment of potato and Arabidopsis bZIP proteins with 1,000 bootstrap replicates using the neighbor-joining ( $\mathrm{NJ}$ ) method. The potato bZIP members together with their Arabidopsis homologs were classified into 11 groups.

(Supplementary Table 4). Among them, motif 1 represents the basic leucine zipper domain, and all the identified bZIP members harbor motif 1 (Supplementary Figure 2). Members of the same group share similar motifs. The members of groups $\mathrm{G}, \mathrm{H}, \mathrm{F}$, and $B$ only contain motif 1 , members of group $S$ contain motifs 1 and 5, group I and group E members harbor motifs 1 and 2, and members of group A harbor motifs 1, 6, 7, 8, and 9. It is worth noting that motifs $3,4,9$, and 10 were highly conserved and only existed in group $\mathrm{D}$, indicating that they were conserved protein domains which may undertake a special function.

\section{Chromosomal Distribution and Duplication Events}

The 49 StbZIP genes were mapped onto 12 potato chromosomes (Figure 3). The most potato bZIP genes (10) were found on chromosome 1, while chromosomes 9,11 , and 12 only harbor one potato $6 Z I P$ gene. It has been reported that when a chromosome region within $200 \mathrm{~kb}$ possesses two or more genes of the same family, these genes are defined to be a gene cluster, and genes sharing an identity of more than $70 \%$ in a cluster are considered to be tandem duplication genes (Holub, 2001). Homology analysis showed that there is one cluster of $b Z I P$ genes on chromosome 1 and chromosome 4. In addition, there are two StbZIP genes (StbZIP04/StbZIP05) that formed a tandem duplication pair.

Furthermore, MCScanX was used to analyze segmental duplication or whole-genome duplication events of the potato bZIP genes. A total of 17 pairs of segmental duplication with 23 StbZIP genes were identified (Figure 4). These results showed that the formation of some StbZIP genes may be caused by duplication events and that these segmental duplication events might play a major role in the evolution of StbZIP genes. All of the tandem and segmental duplication genes are listed in Supplementary Table 5.

The ratio of $\mathrm{Ka} / \mathrm{Ks}$ and the ratio between non-synonymous and synonymous substitutions can be used to estimate whether the selective pressure acts on a protein-coding gene. There are 


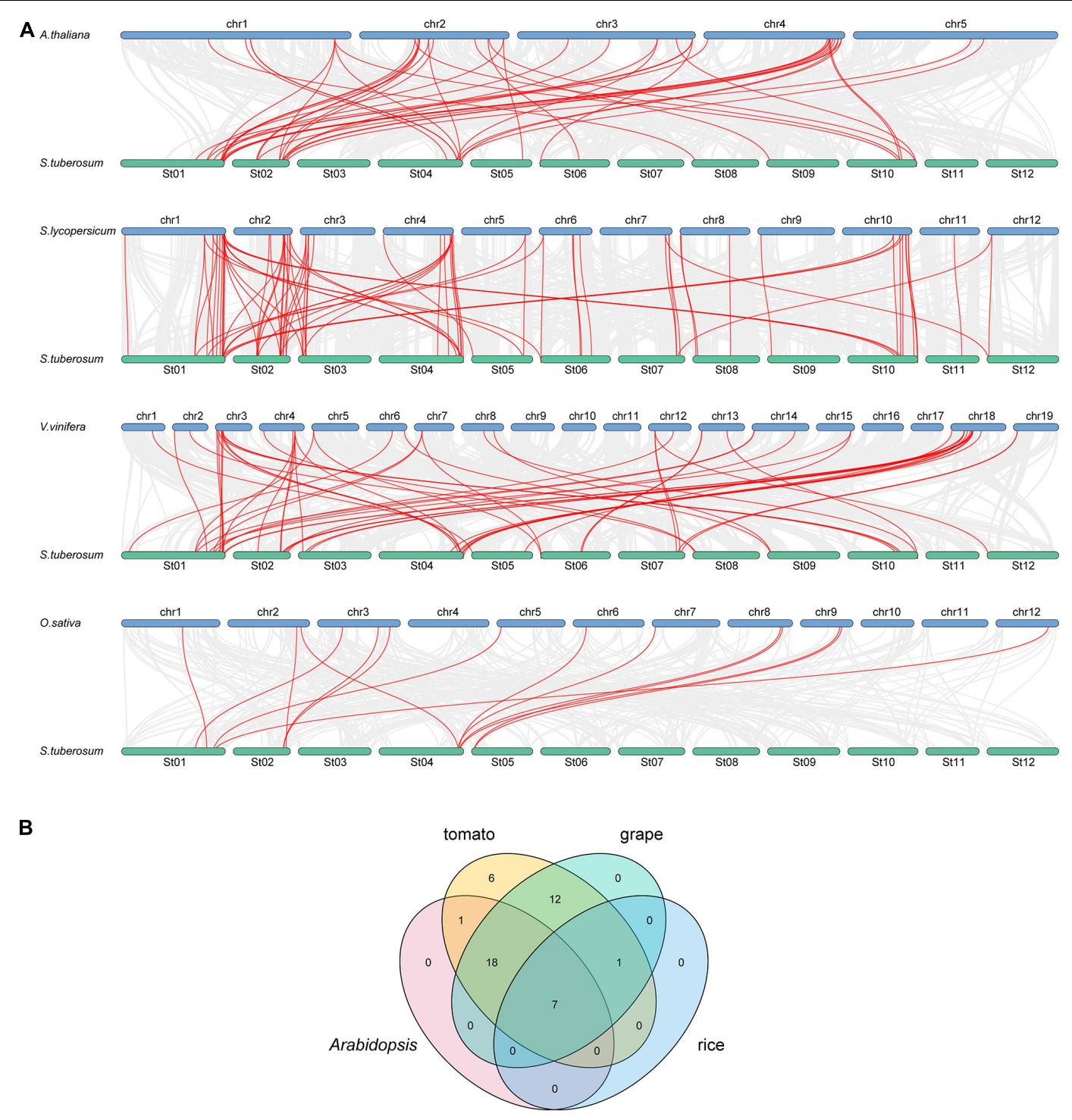

FIGURE 2 | Synteny analysis of bZIP genes between potato and four other representative plant species. (A) The gray line in the background represents the collinear blocks between potato and four other representative species, while the red line exhibit the syntenic bZIP gene pairs. (B) The numbers of bZIP genes that formed syntenic pairs between potato and all the other four selected species which visualized by the Venn plot.

three selection types in evolutionary analysis, including positive selection, neutral selection, and purifying selection. All the Ka/Ks ratios of the 17 segmental duplication pairs were lower than 1 , suggesting that these $b Z I P$ genes may have undergone purifying selective pressure in the process of evolution.

\section{Promoter Analysis of StbZIP Genes}

Several putative cis-elements on promoter regions were identified in the 49 StbZIP gene promoters (Figure 5 and Supplementary Table 6). One or more MYC elements were identified in the promoters of all the StbZIP genes. In addition, hormoneresponsive elements, including $\mathrm{ABRE}$, ERE, and the CGTCA motif, were identified in the promoter regions of some StbZIP genes. These cis-elements are potentially responsive to abscisic acid, ethylene, and methyl jasmonate. Furthermore, 37 of the StbZIP genes contain an anaerobic induction element (ARE), 29 contain at least one WRKY binding site, 27 contain at least one wound-responsive element (WUN motif), 22 contain at least one low-temperature-responsive element (LTR), and 15 of the genes contain at least one stress-responsive element (TC-rich repeats), implying that these StbZIP genes may be involved in various stress responses.

\section{Expression of StbZIP Genes in Different Tissues}

To understand the potential functions of the StbZIP genes, their tissue-specific expression levels were analyzed using RNA-Seq data from the PGSC database. Seven tissues were selected for 

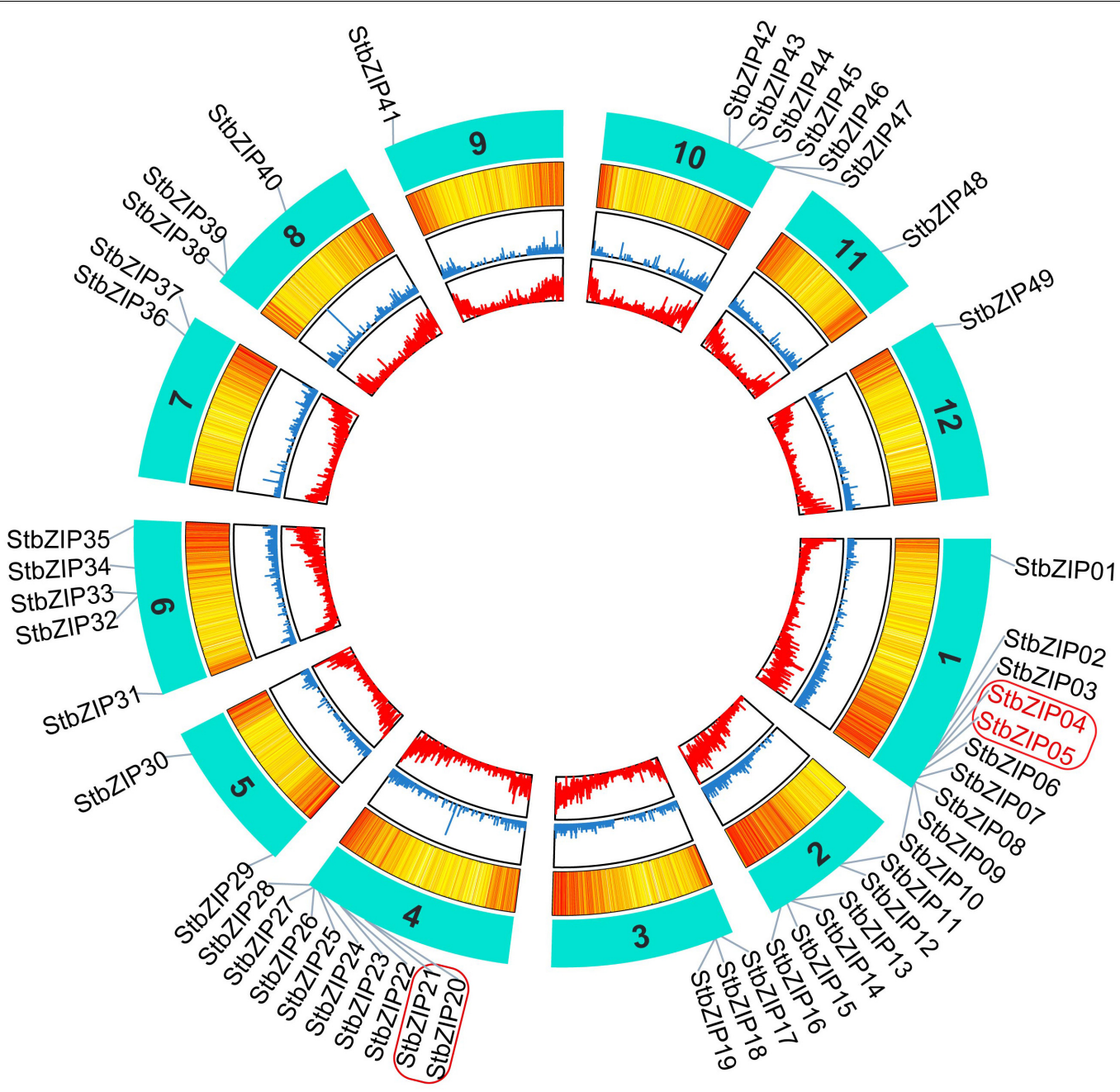

FIGURE 3 | Distribution of StbZIP genes on potato chromosomes. In potato, 49 StbZIP genes were successfully mapped to 12 potato chromosomes. The red box indicate the gene cluster, while the tandem duplication pair is featured by the red color.

analysis, including the root, stem, shoot apex, leaf, flower, young tuber, and mature tuber. Forty-one of the 49 StbZIP genes were expressed in at least one tested tissue, and the transcriptome data were standardized and displayed in R (Figure 6). A number of StbZIP genes were expressed in all the tested tissues, including StbZIP08, StbZIP02, StbZIP26, StbZIP31, and StbZIP43 from group $S$. Other $S t b Z I P$ genes showed tissue-specific expression patterns. The expression of StbZIP04 was only detected in the flower, StbZIP42 was expressed exclusively in the root, and StbZIP17 and StbZIP29 were only expressed in the roots and stems. Furthermore, compared with the other tissues, StbZIP13 and StbZIP37 were specifically upregulated in young tubers.

\section{Expression Patterns of StbZIP Genes in Response to Stress Treatments}

The bZIP genes have been previously reported to be involved in biotic and abiotic stresses (Banerjee and Roychoudhury, 2017). To investigate the roles of StbZIP genes under stresses, their gene expression changes under biotic and abiotic stresses were analyzed. Salt, mannitol, and heat treatments were used for abiotic stresses, and Phytophthora infestans, $\beta$-aminobutyric acid (BABA), and benzothiadiazole (BTH) treatments were employed for biotic stresses. The results indicated that 24 StbZIP genes responded to abiotic stresses (Figure 7A), among which StbZIP25 was induced by salt, mannitol, and heat treatments; StbZIP29 was only highly expressed under heat stress; StbZIP13 and StbZIP17 were highly expressed under salt and heat stresses; and StbZIP11, StbZIP24, and StbZIP47 were significantly induced under salt and drought stresses. Interestingly, the expressions of StbZIP24 and StbZIP47 were suppressed by heat stress. The expression patterns of StbZIP44, StbZIP14, StbZIP42, StbZIP06, StbZIP32, StbZIP15, StbZIP24, and StbZIP47 were similar in response to stresses: induced by salt and drought, while suppressed by heat.

Under biotic stresses, 14 StbZIP genes presented differential expressions when compared with the untreated control (Figure 7B). Among these, only StbZIP02 was upregulated after all three biotic stresses. StbZIP34 and StbZIP28 were found 


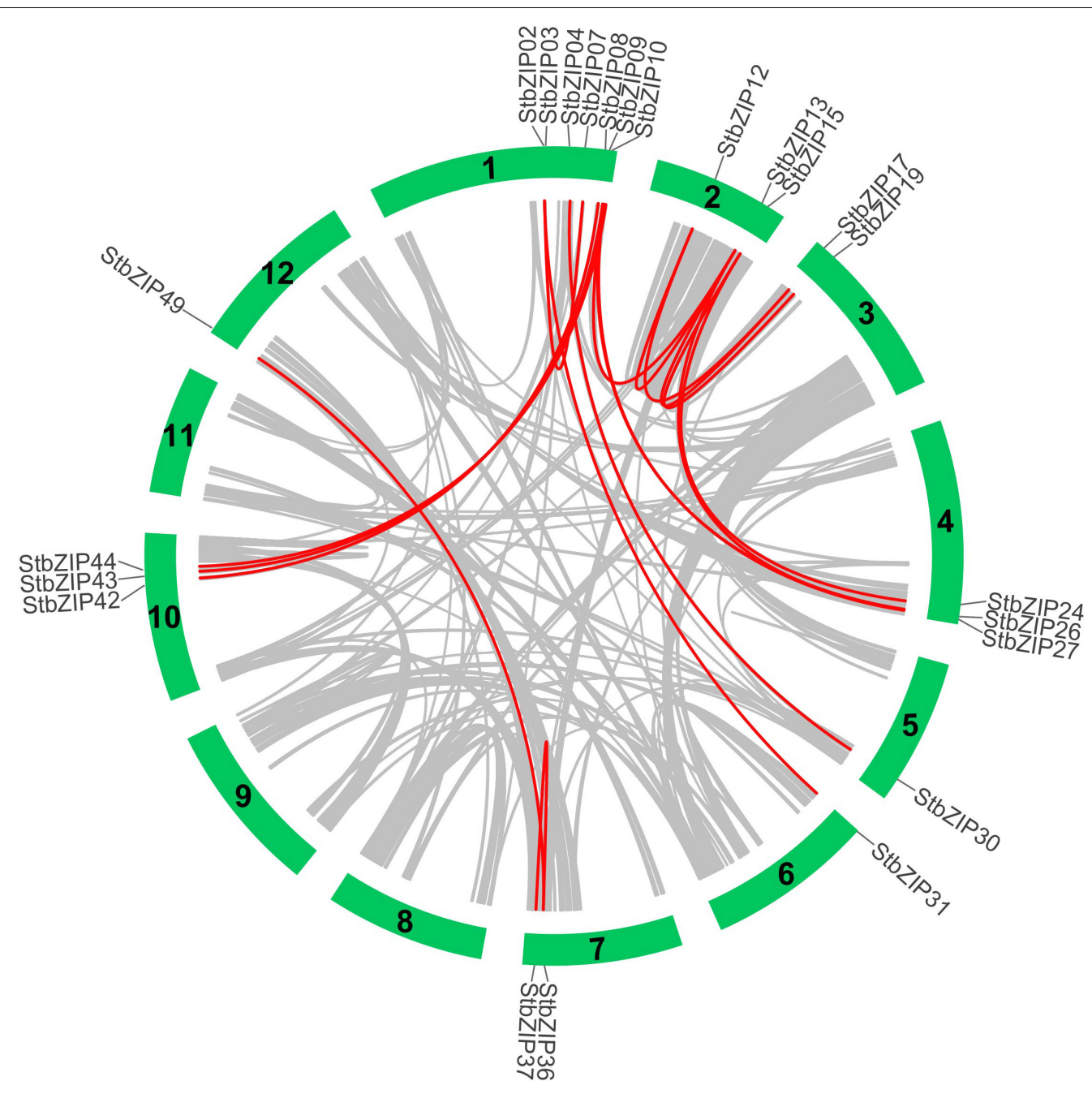

FIGURE 4 | Segmental duplication events and interchromosomal relationships between StbZIP genes. The 31 putative segmental duplication pairs of StbZIP genes were investigated with MCScanX and linked by the colored lines, respectively. The gray lines indicate all putative segmental duplication pairs in the potato genome, while the StbZIP segmental duplication pairs are linked by the red line.

to be upregulated under the stress of BABA. On the contrary, the expressions of StbZIP30, StbZIP03, and StbZIP15 were decreased under BABA stress. StbZIP29 was upregulated after P. infestans inoculation, while StbZIP05 and StbZIP19 exhibited downregulated expressions under this stress.

Furthermore, we found that 32 StbZIP genes were induced under both abiotic and biotic stress conditions (Figure 7C). StbZIP29 was highly induced by $P$. infestans inoculation and under heat stress treatments, and StbZIP15 was upregulated by BTH and in response to salt and drought treatments. Similarly, StbZIP19 responded to all abiotic stresses and BABA treatment (Supplementary Table 7).

\section{Validation of Expression Patterns by qRT-PCR}

qRT-PCR was conducted to verify the RNA-Seq expression patterns of potato bZIP genes under salt stress. As shown in
Figure 8, the expression levels of the selected 15 bZIP genes were all enhanced by the salt treatment, which was consistent with the RNA-Seq data. Among them, the expression levels of eight StbZIP genes kept increasing with the increase of salt treatment time. This included StZIP06, StZIP08, StZIP10, StZIP11, StZIP12, StZIP19, StZIP24, and StZIP25. It is worth noting that StbZIP10 is a close homolog of AtbZIP24, which was reported to enhance salt tolerance via regulating stress-responsive genes (Yang et al., 2009), suggesting that StbZIP10 may also be involved in potato salt stress response. The expression patterns of the other genes were somewhat complicated. For instance, StZIP13 and StZIP32 were highly induced after $1 \mathrm{~h}$ of salt treatment, then decreased after $3 \mathrm{~h}$, and finally increased again after $6 \mathrm{~h}$ treatment. StZIP26 expression was in a similar updown-up pattern, except that it reached the peak after $3 \mathrm{~h}$ of treatment then began to decline. The expressions of StZIP15 and StZIP43 were both suppressed after $1 \mathrm{~h}$ of salt treatment, then increased $6 \mathrm{~h}$ after treatment, suggesting that there might be 


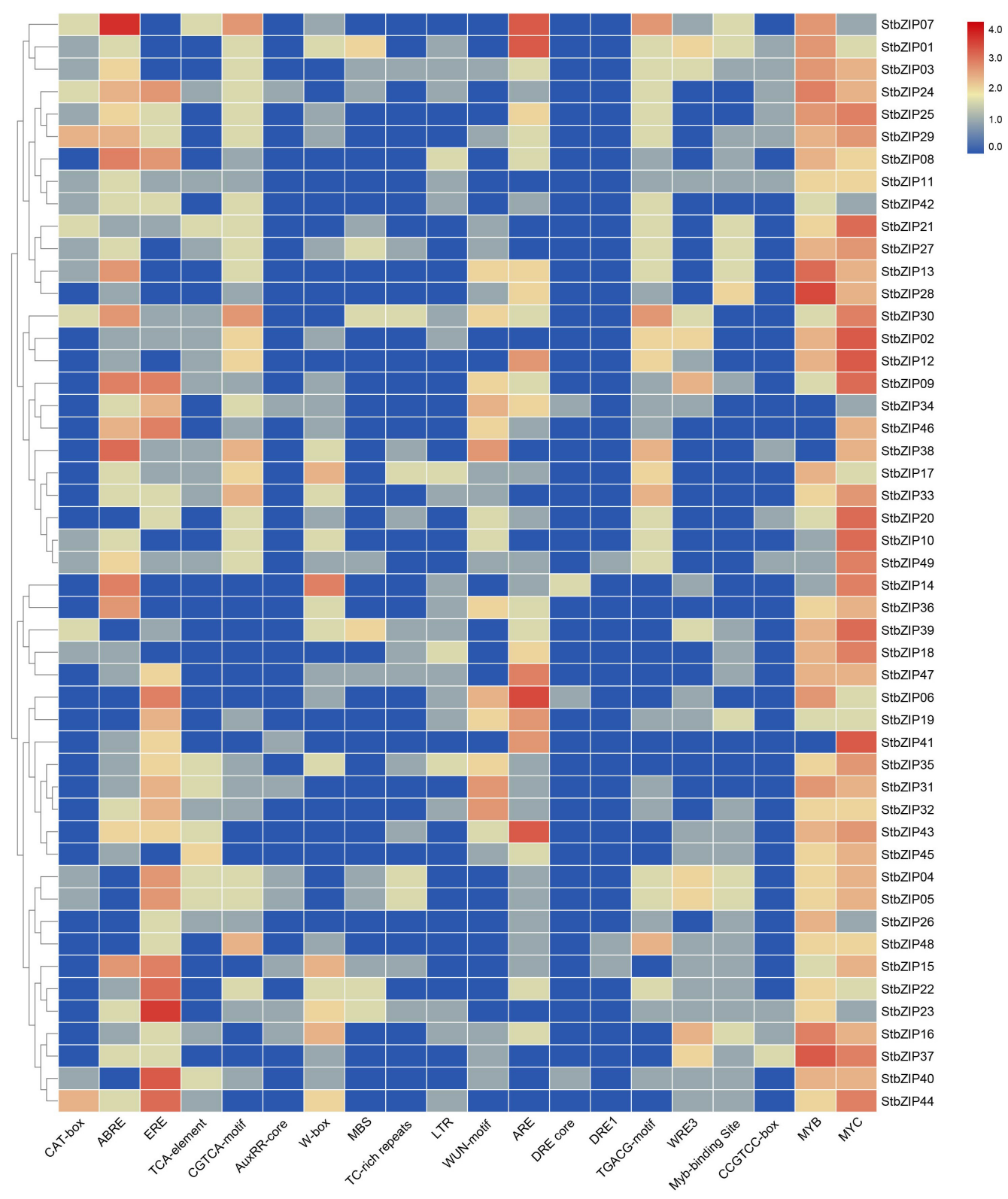

FIGURE 5 | Regulatory elements in the promoter regions of StbZIP genes.

feedback activation mechanisms involved in their response to salt stress treatments.

\section{Subcellular Location and Transactivation Activity Assays}

In order to analyze the subcellular localization of the StbZIP proteins, the group A member StbZIP25 was selected as a representative to perform the experiment. The 35S:StbZIP25-GFP recombinant construct and the 35S:GFP control were transiently expressed in $N$. benthamiana and the subcellular localization of the GFP signal was observed by confocal microscopy. As a result, the green fluorescence of the 35S:GFP control in tobacco leaf epidermal cells was distributed on plasma membranes and in cytoplasm, whereas the StbZIP25-GFP fusion proteins were only found in the nucleus, indicating that the StbZIP25 protein was localized in the nucleus (Figure 9A).

To investigate whether StbZIP25 acts as a transcriptional activator, transcriptional activation assays were performed using the StbZIP25 full-length CDS (Figure 9B). Transformed AH109 yeast cells selected on SD/-Trp media were transferred to SD/Trp/X-gal media. Activation of the reporter gene in the yeast cells was determined by assays of the $\beta$-galactosidase activities with $\mathrm{X}$-gal as the substrate. The yeast cells containing the full-length StbZIP25 (GAL4BD-StbZIP25) were blue on the SD/-Trp/X-gal media, whereas the cells with the pBridge empty vector was 


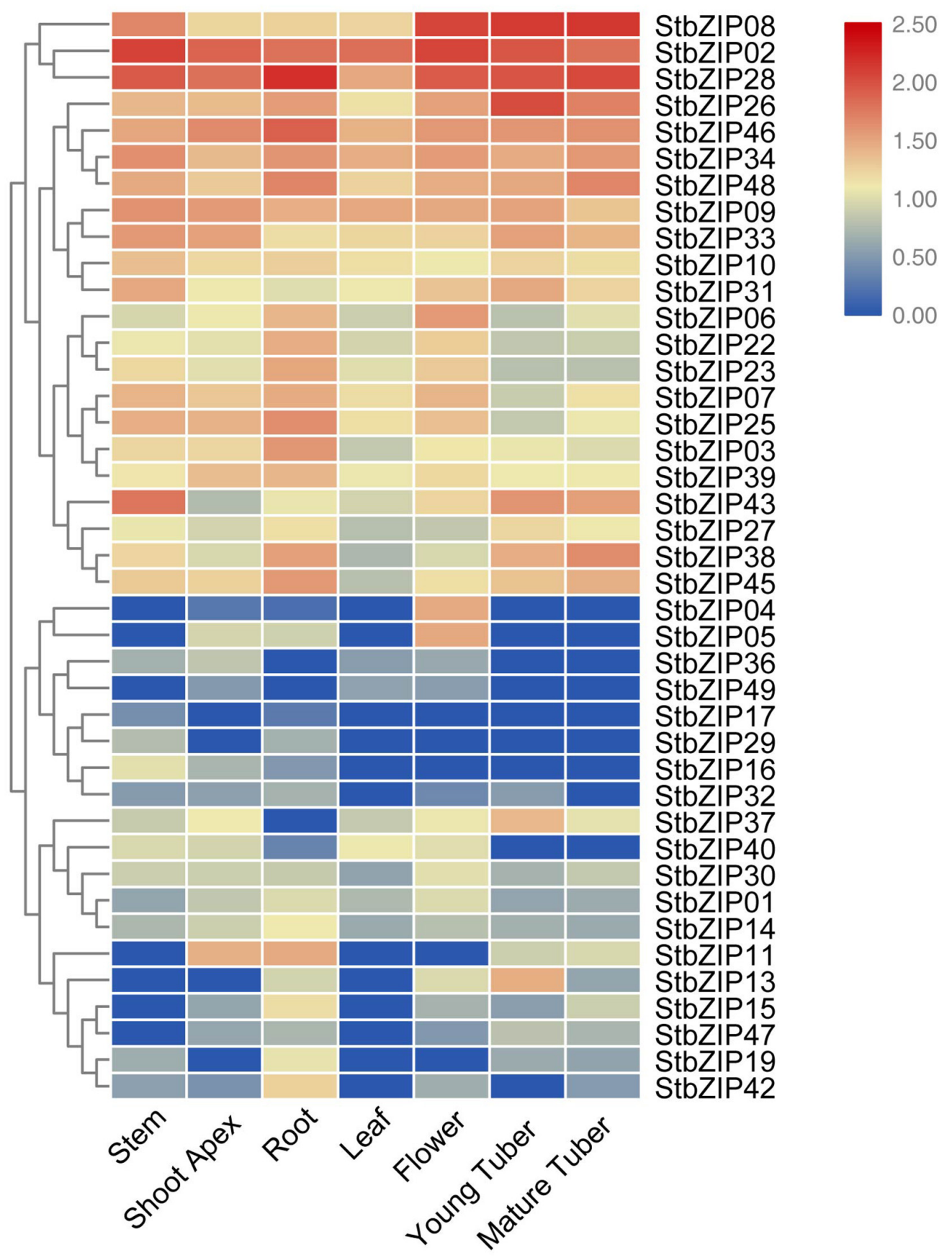

FIGURE 6 | Expression patterns of the StbZIP genes in the tested tissues. The expression pattern data were retrieved from transcriptome data and visualized by TBtools.

white (Figure 9B), indicating that StbZIP25 functioned as a transcriptional activator.

\section{Overexpression of StbZIP25 Enhanced Salt Tolerance in Arabidopsis}

To further explore the function of the salt-responsive gene StbZIP25 in abiotic stresses, transgenic Arabidopsis plants overexpressing StbZIP25 were generated. Five T0 transgenic lines were verified by PCR using genomic DAN templates. The homozygous T3 transgenic lines were selected and the expression level of StbZIP25 was analyzed using qRT-PCR. Two overexpression lines (OE2 and OE5) with higher expression levels of StbZIP25 were selected for further analysis (Supplementary Figure 3). The root length of plants grown on 1/2 MS medium with $100 \mathrm{mM} \mathrm{NaCl}$ was measured to assess salt tolerance 

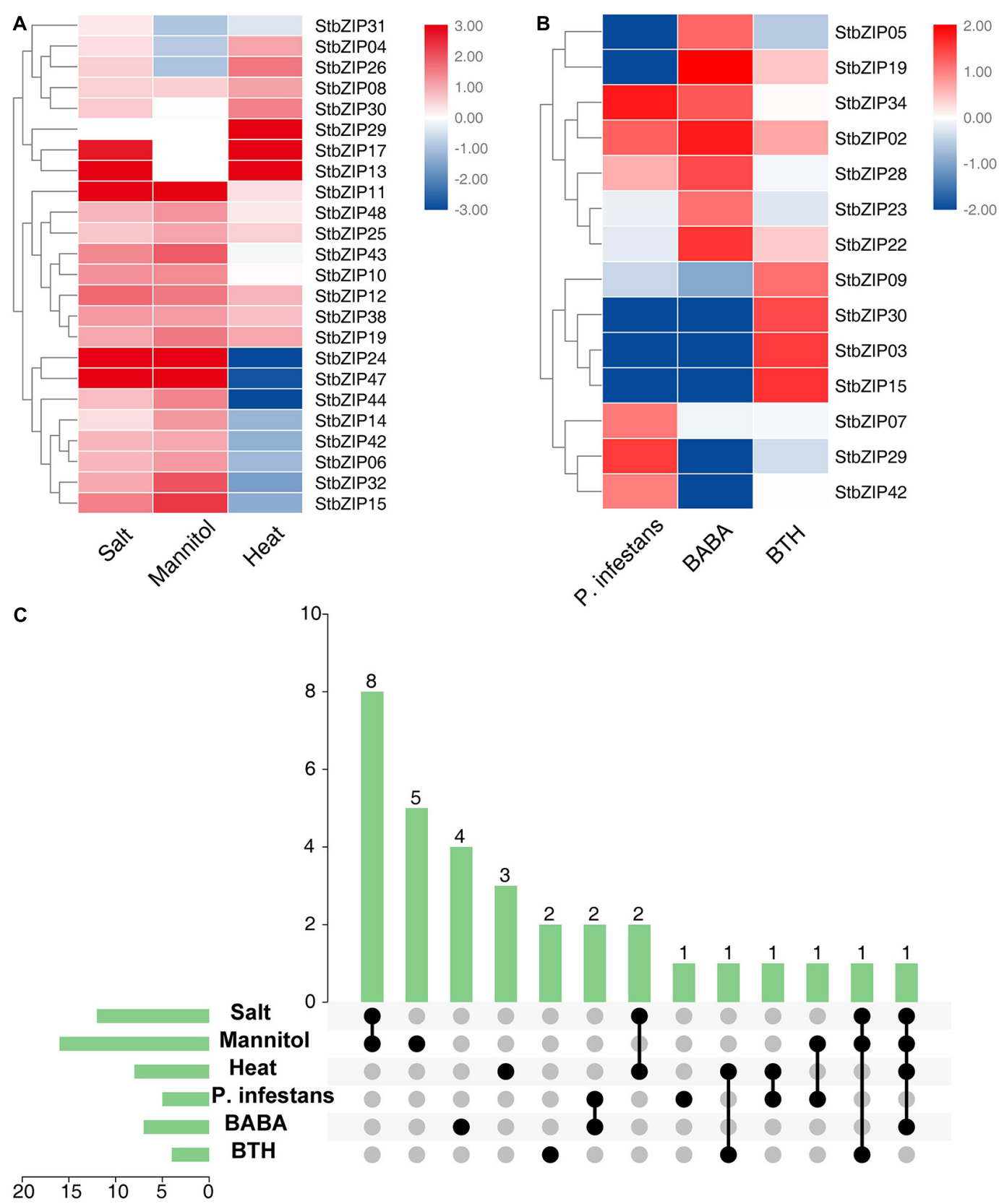

FIGURE 7 | The expression patterns of StbZIP genes under abiotic and biotic stress treatments. (A) Relative expression ratios of the abiotic stress treatments. (B) Relative expression ratios of the biotic stress treatments. (C) Summarized information of the stress-induced StbZIP genes. The relative expression ratios of the abiotic and biotic stress treatments were calculated relative to the untreated control and then the significantly induced gene was defined to possess a log 2 relative expression ratio $\geq 1$ under one of the stress treatments. The red, white, and blue colors represent the upregulated, unaltered, and downregulated expressions, respectively. The vertical axis and vertical bars represent the number of genes responding to stresses. The points represent the number of genes responding to one or more stresses.

(Figure 10). WT and StbZIP25-overexpressing Arabidopsis plants showed no significant difference in root length under normal conditions. However, after 3 weeks of growing on $100 \mathrm{mM} \mathrm{NaCl}$ media, the transgenic plants displayed a longer root phenotype compared with the WT. Taken together, the results revealed that the overexpression of StbZIP25 enhanced the salt tolerance in transgenic Arabidopsis.

\section{DISCUSSION}

The bZIP gene family has been reported to be one of the largest transcription factor families in plants that functions in plant development, metabolism, and stress responses (Wang et al., 2015; Droge-Laser et al., 2018). Furthermore, bZIP proteins have been identified in a number of plant species, including 

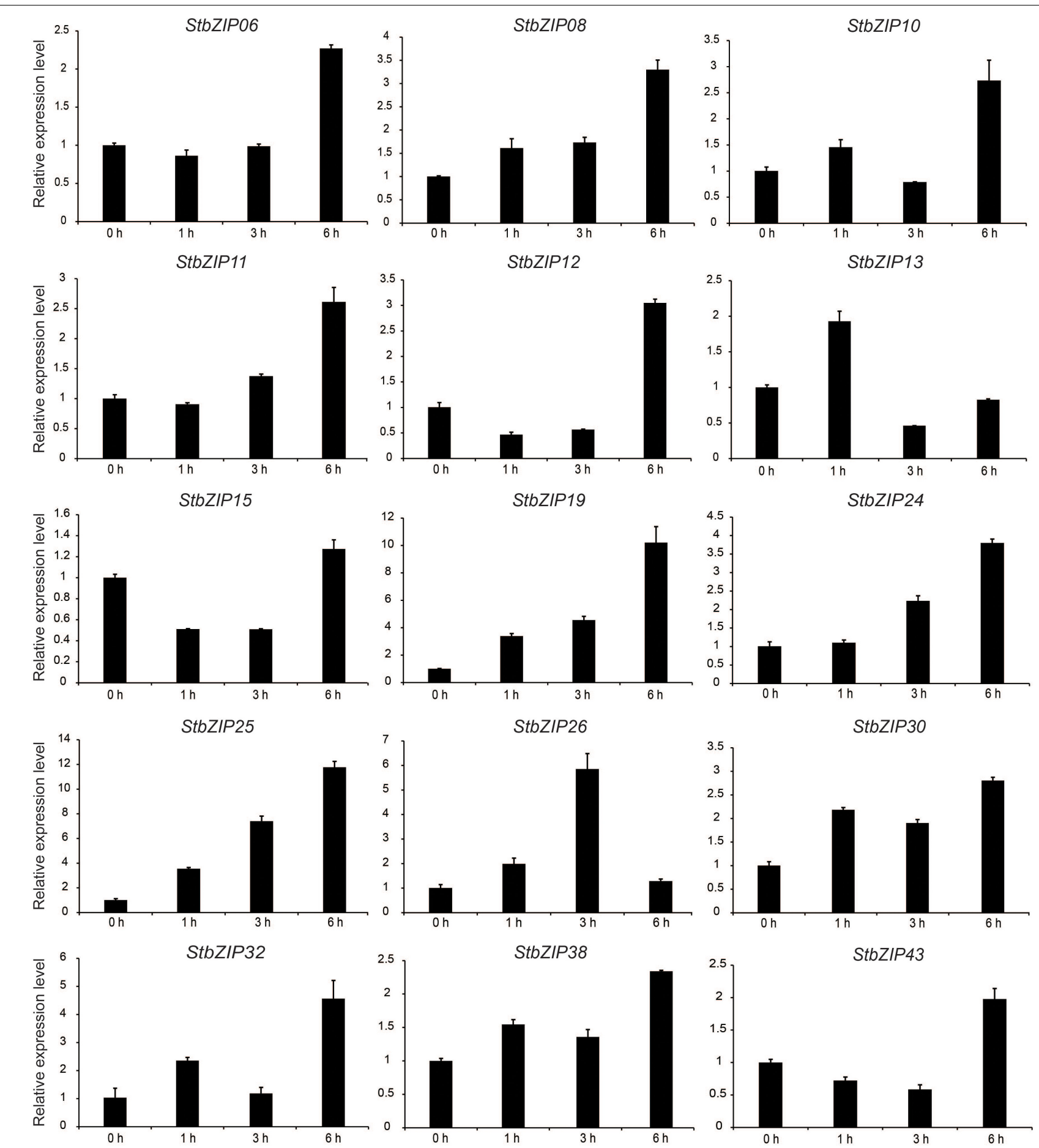

FIGURE 8 | The expression patterns of selected StbZIP genes in response to salt stress treatments, which were calculated as folds relative to the untreated control.

Arabidopsis (Jakoby et al., 2002), maize (Wei et al., 2012), rice (Nijhawan et al., 2008), sorghum (Wang et al., 2011), and wheat (Li et al., 2015). In this study, a total of 49 bZIP genes were identified from the potato genome by using BLASTP searches. The number of StbZIP genes was lower than the number of bZIP genes in Arabidopsis (75 genes) (Jakoby et al., 2002) and was similar to that in grapevine (55 genes) (Liu et al., 2014) and in castor bean (49 genes) (Jin et al., 2014). The potential potato $b Z I P$ genes were studied through analyses of the phylogeny, gene structure, motif organization, synteny, chromosomal distribution, duplication events, cis-elements, and expression profiles. 


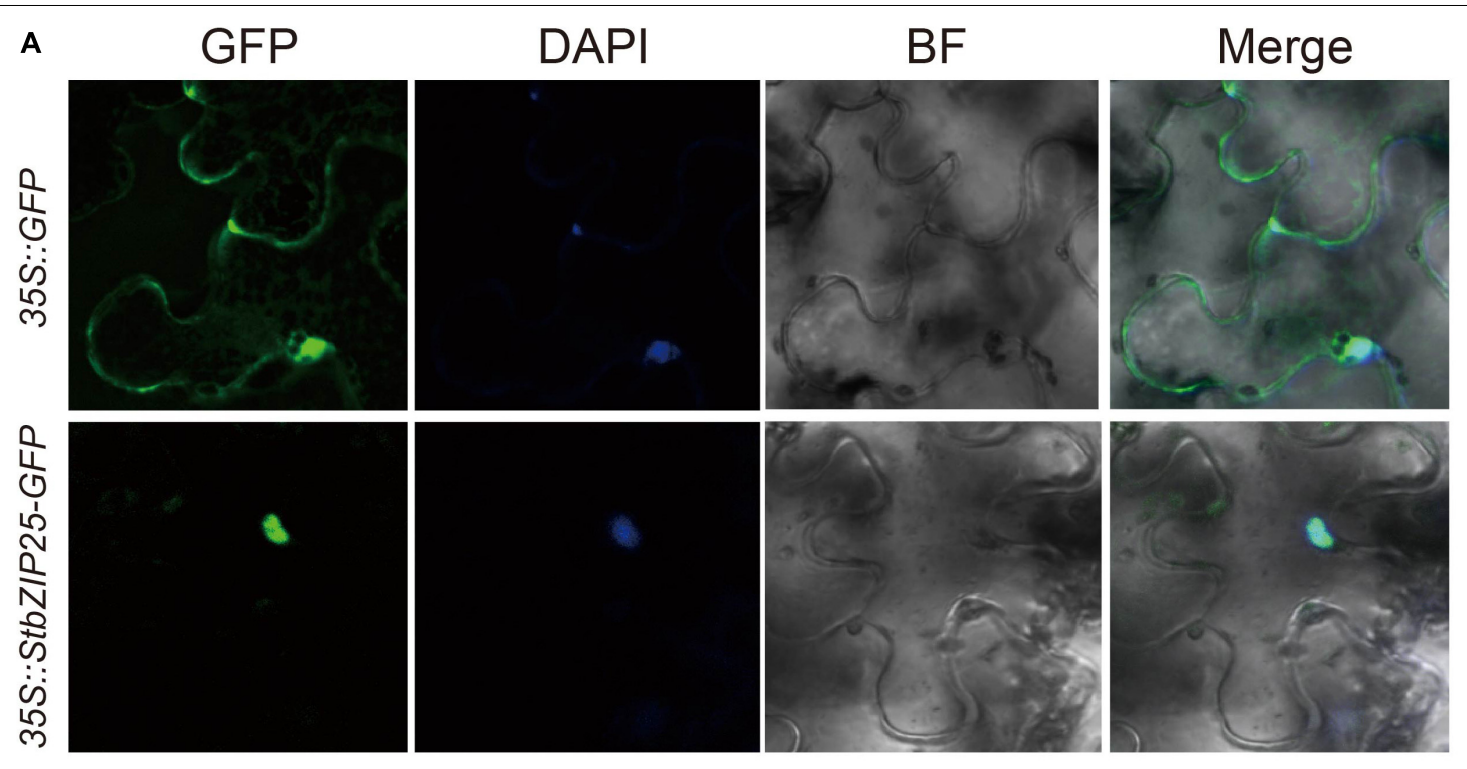

B

pBridge

GAL4BD

\section{GAL4BD-StbZIP25 GAL4BD}

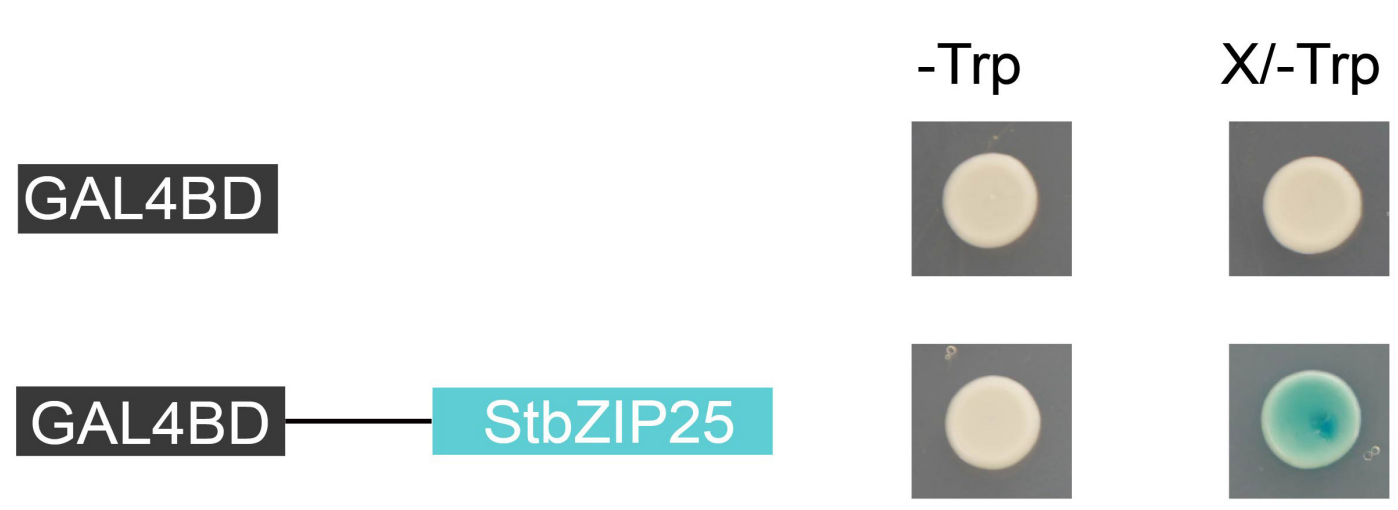

FIGURE 9 | The subcellular localization and transactivation assays of StbZIP25. (A) The StbZIP25-GFP fusion construct and the GFP gene driven by the CaMV-35S promoter were transiently expressed into tobacco. DAPI (dye 4,6-diamidino-2-phenylindole) staining indicated the nucleus. (B) Transactivation activity assay of StbZIP25 in yeast strain AH109. $\beta$-galactosidase activities against X-gal were detected on SD/-Trp media.

Tandem and segmental duplication play key roles in the extension of a gene family (Vision et al., 2000; Cannon et al., 2004). In this study, 17 segmental duplication pairs were found in the potato bZIP gene family, whereas only one tandem duplication pair was identified (Figures 3, 4), suggesting that the expansion of the StbZIP gene family in potato was mainly originated from segmental duplication. The $\mathrm{Ka} / \mathrm{Ks}$ ratios of all these duplicated pairs were lower than 1 (Supplementary Table 5), suggesting that these duplicated StbZIP genes might have been purifying selected and maintained conserved functions in evolution. For example, StbZIP04 and StbZIP05, as a tandem duplication pair, were both highly expressed in flower tissues (Figure 6).
A number of $b Z I P$ genes have been reported to be involved in controlling Arabidopsis development. In group $\mathrm{C}$, AtbZIP9/BZO2H2 has been reported to alter vascular development in roots (Silveira et al., 2007). StbZIP38 was clustered together with AtbZIP9 in group C, and this gene showed a high level of expression in roots (Figure 6), implying that StbZIP38 may also be involved in root development. In group I, AtbZIP29 was reported to function in leaf and root development (van Leene et al., 2016). Its homolog, StbZIP09, was found to be highly expressed in both roots and leaves, which suggested that AtbZIP29 and StbZIP09 might have similar biological functions. AtbZIP44 in group $S$ was reported to participate in regulating seed germination 

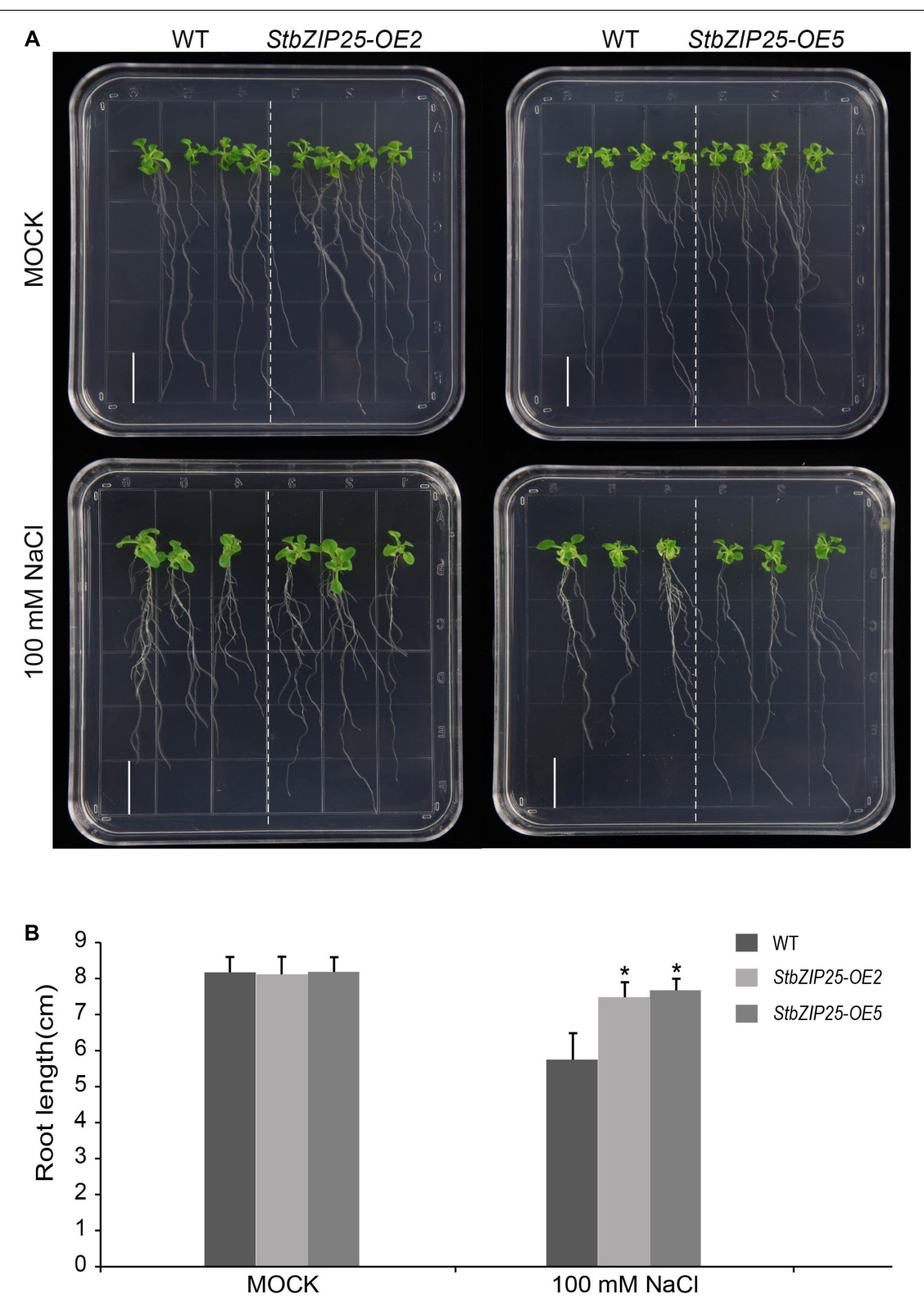

FIGURE 10 | Effects of salt stress treatments on the root growth of the StbZIP25 gene overexpressed in Arabidopsis. (A) Primary root lengths of the wild-type and StbZIP25 overexpression lines under salt treatments in transgenic Arabidopsis. (B) Quantification of the primary root lengths under normal condition and 100 mM $\mathrm{NaCl}$ treatments. Bar, $1.5 \mathrm{~cm}$. The data were retrieved from three biological replicates. WT, wild type. Data are the mean $\pm \mathrm{SD}$ of three biological repeats. ${ }^{\star} p<0.05$ ( $t$ tests).

(Iglesias Fernandez et al., 2013). Although gene expression in seeds was not examined in this study, a close homolog of AtZIP44 in potato, StbZIP08, was found to be highly expressed in the flower (Figure 6).

Increasing evidence indicates that bZIP family members can function as regulators in plants' responses to abiotic stresses. AtbZIP24 in group F, which has been reported to be involved in regulating abiotic stress responses (Yang et al., 2009), was clustered together with StbZIP10 and StbZIP44. Interestingly, StbZIP10 and StbZIP44 were found to be induced by salt/drought stresses (Figure 7A). In addition, the $\mathrm{ABF}$ subfamily members of bZIP proteins are involved in ABA/stress responses (Banerjee and Roychoudhury, 2017). The overexpression of AtbZIP36/ABF2 altered ABA sensitivity, dehydration tolerance, and the expression levels of ABA/stressregulated genes (Kim et al., 2010). The promoter of the AtbZIP36/ABF2 homologous gene StbZIP25 contains the ciselement ABRE (Figure 5), suggesting that it may also be related to ABA signaling and stress responses. In addition, salt and drought stress treatments can significantly induce the expression of StbZIP25 (Figures 7A, 8), suggesting that it may participate in the response to plant salt and drought stresses. Further 
experiments indicated that the overexpression of StbZIP25 can confer salt tolerance in transgenic Arabidopsis (Figure 10), supporting the role of StbZIP in stress response and the potential functional conservation of plant $b Z I P$ genes between species. The StbZIP25 protein was shown to be located in the nucleus and showed transcriptional activation activities (Figure 9), indicating that StbZIP25 acts as a transcription activator to regulate gene expression in response to stresses. StbZIP25 was chosen as a representative of StbZIP members showing stress-responsive expressions which could potentially function as regulators of stress responses. The results of StbZIP25 overexpression conferred salt tolerance in Arabidopsis (Figure 10), supporting the potential roles of more StbZIP proteins in stress responses.

\section{CONCLUSION}

In this study, a systematic study of the potato bZIP gene family was carried out, including the identification of the potato bZIP family members, analyses on evolutionary relationship, chromosome locations, and the expression patterns of StbZIP genes. The results suggest that the StbZIP genes may be important for regulating plant responses to abiotic/biotic stresses and development. Notably, AtbZIP36/ABF2 has been reported to participate in regulating the response to abiotic stresses. Its potato homolog StbZIP25 was induced by salt and drought stresses and was able to enhance salt tolerance in transgenic Arabidopsis. Overall, the results from this study will help in further investigations of the function of potato $b Z I P$ genes.

\section{DATA AVAILABILITY STATEMENT}

The original contributions presented in the study are included in the article/Supplementary Material, further inquiries can be directed to the corresponding authors.

\section{AUTHOR CONTRIBUTIONS}

$\mathrm{XL}$ and YG conceived this research, designed the experiments, and drafted the manuscript. QW and CG conducted the research

\section{REFERENCES}

Bailey, T. L., Boden, M., Buske, F. A., Frith, M., Grant, C. E., Clementi, L., et al. (2009). MEME SUITE: tools for motif discovery and searching. Nucleic Acids Res. 37, W202-W208. doi: 10.1093/nar/gkp335

Banerjee, A., and Roychoudhury, A. (2017). Abscisic-acid-dependent basic leucine zipper (bZIP) transcription factors in plant abiotic stress. Protoplasma 254, 3-16. doi: 10.1007/s00709-015-0920-4

Beitz, E. (2000). TEXshade: shading and labeling of multiple sequence alignments using LATEX2 epsilon. Bioinformatics 16, 135-139. doi: 10.1093/ bioinformatics/16.2.135

Bensmihen, S., Rippa, S., Lambert, G., Jublot, D., Pautot, V., Granier, F., et al. (2002). The homologous ABI5 and EEL transcription factors function antagonistically to fine-tune gene expression during late embryogenesis. Plant Cell 14, 1391-1403. doi: 10.1105/tpc.000869

Cannon, S. B., Mitra, A., Baumgarten, A., Young, N. D., and May, G. (2004). The roles of segmental and tandem gene duplication in the evolution of large gene and participated in drafting the manuscript. ZL, JS, DW, and LX assisted in data collection and analysis. All authors contributed to the article and approved the submitted version.

\section{FUNDING}

This work was supported by the National Natural Science Foundation of China (31571494), the Agricultural Science and Technology Innovation Program (ASTIP-TRIC02), and the Fundamental Research Funds for Central Non-Profit Scientific Institution (Y2017JC27).

\section{SUPPLEMENTARY MATERIAL}

The Supplementary Material for this article can be found online at: https://www.frontiersin.org/articles/10.3389/fpls.2021. 637343/full\#supplementary-material

Supplementary Figure 1 | Sequence logos of the basic region of bZIP members from potato (A) and Arabidopsis (B)

Supplementary Figure 2 | The motif and gene structure organizations of StbZIP members.

Supplementary Figure 3 | (A) Five T0 transgenic lines were verified by normal PCR using genomic DNA templates. (B) The expression levels of StbZIP25 gene in wild type and two overexpression lines. The ratios of gene expression were calculated relative to the wild type.

Supplementary Table 1 | The qRT-PCR primers used in this study.

Supplementary Table 2 | The detail information of identified potato bZIP family members.

Supplementary Table 3 | The syntenic pairs between potato and other four plant species.

Supplementary Table 4 | The putative conserved motifs in StbZIP proteins.

Supplementary Table $\mathbf{5}$ | The detail information of segmental and tandem duplication gene pairs.

Supplementary Table 6 | Regulatory elements in the promoter regions of StbZIP genes.

Supplementary Table 7 | The detail information of the induced StbZIP gene under various stress treatments.

families in Arabidopsis thaliana. BMC Plant Biol. 4:10. doi: 10.1186/1471-22294- 10

Chen, C., Chen, H., Zhang, Y., Thomas, H. R., Frank, M. H., He, Y., et al. (2020). TBtools: An integrative toolkit developed for interactive analyses of big biological data. Mol. Plant 13, 1194-1202. doi: 10.1016/j.molp.2020. 06.009

Dietrich, K., Weltmeier, F., Ehlert, A., Weiste, C., Stahl, M., Harter, K., et al. (2011). Heterodimers of the Arabidopsis transcription factors bZIP1 and bZIP53 reprogram amino acid metabolism during low energy stress. Plant Cell 23, 381-395. doi: 10.1105/tpc.110.0 75390

Droge-Laser, W., Snoek, B. L., Snel, B., and Weiste, C. (2018). The Arabidopsis bZIP transcription factor family-an update. Curr. Opin. Plant Biol. 45, 36-49. doi: 10.1016/j.pbi.2018.05.001

El Gebali, S., Mistry, J., Bateman, A., Eddy, S. R., Luciani, A., Potter, S. C., et al. (2019). The Pfam protein families database in 2019. Nucleic Acids Res. 47, D427-D432. doi: 10.1093/nar/gky995 
Gan, S., and Amasino, R. M. (1997). Making sense of senescence (molecular genetic regulation and manipulation of leaf senescence). Plant Physiol. 113, 313-319. doi: 10.1104/pp.113.2.313

Gao, S., Gao, J., Zhu, X., Song, Y., Li, Z., Ren, G., et al. (2016). ABF2, ABF3, and $\mathrm{ABF} 4$ promote $\mathrm{ABA}$-mediated chlorophyll degradation and leaf senescence by transcriptional activation of chlorophyll catabolic genes and senescenceassociated genes in Arabidopsis. Mol. Plant 9, 1272-1285. doi: 10.1016/j.molp. 2016.06.006

Gibalova, A., Steinbachova, L., Hafidh, S., Blahova, V., Gadiou, Z., Michailidis, C., et al. (2017). Characterization of pollen-expressed bZIP protein interactions and the role of ATbZIP18 in the male gametophyte. Plant Reprod. 30, 1-17. doi: 10.1007/s00497-016-0295-5

Guo, Y., and Gan, S. S. (2014). Translational researches on leaf senescence for enhancing plant productivity and quality. J. Exp. Bot. 65, 3901-3913. doi: 10. 1093/jxb/eru248

Holub, E. B. (2001). The arms race is ancient history in Arabidopsis, the wildflower. Nat. Rev. Genet. 2, 516-527. doi: 10.1038/35080508

Hu, B., Jin, J., Guo, A. Y., Zhang, H., Luo, J., and Gao, G. (2015). GSDS 2.0: an upgraded gene feature visualization server. Bioinformatics 31, 1296-1297. doi: 10.1093/bioinformatics/btu817

Iglesias Fernandez, R., Barrero Sicilia, C., Carrillo Barral, N., Onate Sanchez, L., and Carbonero, P. (2013). Arabidopsis thaliana bZIP44: a transcription factor affecting seed germination and expression of the mannanase-encoding gene AtMAN7. Plant J. 74, 767-780. doi: 10.1111/tpj.12162

Izawa, T., Foster, R., and Chua, N. H. (1993). Plant bZIP protein DNA binding specificity. J. Mol. Biol. 230, 1131-1144. doi: 10.1006/jmbi.1993.1230

Jakoby, M., Weisshaar, B., Droge-Laser, W., Vicente-Carbajosa, J., Tiedemann, J., Kroj, T., et al. (2002). bZIP transcription factors in Arabidopsis. Trends Plant Sci. 7, 106-111. doi: 10.1016/s1360-1385(01)02223-3

Jin, Z., Xu, W., and Liu, A. (2014). Genomic surveys and expression analysis of bZIP gene family in castor bean (Ricinus communis L.). Planta 239, 299-312. doi: 10.1007/s00425-013-1979-9

Johnson, L. S., Eddy, S. R., and Portugaly, E. (2010). Hidden Markov model speed heuristic and iterative HMM search procedure. BMC Bioinformatics 11:431. doi: 10.1186/1471-2105-11-431

Katoh, K., Rozewicki, J., and Yamada, K. D. (2019). MAFFT online service: multiple sequence alignment, interactive sequence choice and visualization. Brief. Bioinform. 20, 1160-1166. doi: 10.1093/bib/bbx108

Kim, S., Kang, J. Y., Cho, D. I., Park, J. H., and Kim, S. Y. (2010). ABF2, an ABRE-binding bzip factor, is an essential component of glucose signaling and its overexpression affects multiple stress tolerance. Plant J. 40, 75-87. doi: 10 . 1111/j.1365-313X.2004.02192.x

Landschulz, W. H., Johnson, P. F., and McKnight, S. L. (1988). The leucine zipper: a hypothetical structure common to a new class of DNA binding proteins. Science 240, 1759-1764. doi: 10.1126/science.3289117

Letunic, I., and Bork, P. (2018). 20 years of the SMART protein domain annotation resource. Nucleic Acids Res. 46, D493-D496. doi: 10.1093/nar/ gkx922

Li, X., Ahmad, S., Ali, A., Guo, C., Li, H., Yu, J., et al. (2019a). Characterization of somatic embryogenesis receptor-like kinase 4 as a negative regulator of leaf senescence in Arabidopsis. Cells 8, 50. doi: 10.3390/cells8010050

Li, X., Ahmad, S., Guo, C., Yu, J., Cao, S., Gao, X., et al. (2018). Identification and characterization of LRR-RLK family genes in potato reveal their involvement in peptide signaling of cell fate decisions and biotic/abiotic stress responses. Cells 7:120. doi: 10.3390/cells7090120

Li, X., Gao, S., Tang, Y., Li, L., Zhang, F., Feng, B., et al. (2015). Genome-wide identification and evolutionary analyses of bZIP transcription factors in wheat and its relatives and expression profiles of anther development related TabZIP genes. BMC Genomics 16:976. doi: 10.1186/s12864-015-2196-7

Li, X., Guo, C., Ahmad, S., Wang, Q., Yu, J., Liu, C., et al. (2019b). Systematic analysis of MYB family genes in potato and their multiple roles in development and stress responses. Biomolecules 9, 317. doi: 10.3390/genes 9050260

Librado, P., and Rozas, J. (2009). DnaSP v5: a software for comprehensive analysis of DNA polymorphism data. Bioinformatics 25, 1451-1452. doi: 10.1093/ bioinformatics/btp 187

Liu, C. C., Chi, C., Jin, L. J., Zhu, J., Yu, J. Q., and Zhou, Y. H. (2018). The bZip transcription factor HY5 mediates CRY1a-induced anthocyanin biosynthesis in tomato. Plant Cell Environ. 41, 1762-1775. doi: 10.1111/pce.13171
Liu, J., Chen, N., Chen, F., Cai, B., Dal Santo, S., Tornielli, G. B., et al. (2014). Genome-wide analysis and expression profile of the bZIP transcription factor gene family in grapevine (Vitis vinifera). BMC Genomics 15:281. doi: 10.1186/ 1471-2164-15-281

Liu, J. X., Srivastava, R., and Howell, S. H. (2008). Stress-induced expression of an activated form of AtbZIP17 provides protection from salt stress in Arabidopsis. Plant Cell Environ. 31, 1735-1743. doi: 10.1111/j.1365-3040.2008. 01873.x

Lozano Sotomayor, P., Chavez Montes, R. A., Silvestre Vano, M., Herrera Ubaldo, H., Greco, R., Pablo Villa, J., et al. (2016). Altered expression of the bZIP transcription factor DRINK ME affects growth and reproductive development in Arabidopsis thaliana. Plant J. 88, 437-451. doi: 10.1111/tpj.13264

Muniz Garcia, M. N., Giammaria, V., Grandellis, C., Tellez Inon, M. T., Ulloa, R. M., and Capiati, D. A. (2012). Characterization of StABF1, a stress-responsive bZIP transcription factor from Solanum tuberosum L. that is phosphorylated by StCDPK2 in vitro. Planta 235, 761-778. doi: 10.1007/s00425-0111540-7

Nijhawan, A., Jain, M., Tyagi, A. K., and Khurana, J. P. (2008). Genomic survey and gene expression analysis of the basic leucine zipper transcription factor family in rice. Plant Physiol. 146, 333-350. doi: 10.1104/pp.107.112821

Niu, X., Renshaw-Gegg, L., Miller, L., and Guiltinan, M. J. (1999). Bipartite determinants of DNA-binding specificity of plant basic leucine zipper proteins. Plant Mol. Biol. 41, 1-13. doi: 10.1023/a:1006206011502

Qu, L. J., and Zhu, Y. X. (2006). Transcription factor families in Arabidopsis: major progress and outstanding issues for future research. Curr. Opin. Plant Biol. 9, 544-549. doi: 10.1016/j.pbi.2006.07.005

Quevillon, E., Silventoinen, V., Pillai, S., Harte, N., Mulder, N., Apweiler, R., et al. (2005). InterProScan: protein domains identifier. Nucleic Acids Res. 33, W116-W120. doi: 10.1093/nar/gki442

Rolly, N. K., Imran, Q. M., Lee, I. J., and Yun, B. W. (2020). Salinity stress-mediated suppression of expression of salt overly sensitive signaling pathway genes suggests negative regulation by AtbZIP62 transcription factor in Arabidopsis thaliana. Int. J. Mol. Sci. 21, 1726. doi: 10.3390/ijms21051726

Roychoudhury, A., and Paul, A. (2012). "Abscisic acid-inducible genes during salinity and drought stress," in Advances in Medicine and Biology, ed. L. V. Berhardt (New York, NY: Nova Publishers), 1-78.

Shin, D. H., Choi, M., Kim, K., Bang, G., Cho, M., Choi, S. B., et al. (2013). HY5 regulates anthocyanin biosynthesis by inducing the transcriptional activation of the MYB75/PAP1 transcription factor in Arabidopsis. FEBS Lett. 587, 15431547. doi: 10.1016/j.febslet.2013.03.037

Silveira, A. B., Gauer, L., Tomaz, J. P., Cardoso, P. R., Carmello-Guerreiro, S., and Vincentz, M. (2007). The Arabidopsis AtbZIP9 protein fused to the VP16 transcriptional activation domain alters leaf and vascular development. Plant Sci. 172, 1148-1156. doi: 10.1016/j.plantsci.2007.03.003

Singh, A. K., Sharma, V., Pal, A. K., Acharya, V., and Ahuja, P. S. (2013). Genomewide organization and expression profiling of the NAC transcription factor family in potato (Solanum tuberosum L.). DNA Res. 20, 403-423. doi: 10.1093/ dnares/dst019

Smykowski, A., Zimmermann, P., and Zentgraf, U. (2010). G-Box binding factor1 reduces CATALASE2 expression and regulates the onset of leaf senescence in Arabidopsis. Plant Physiol. 153, 1321-1331. doi: 10.1104/pp.110. 157180

Sun, X., Li, Y., Cai, H., Bai, X., Ji, W., Ding, X., et al. (2012). The Arabidopsis AtbZIP1 transcription factor is a positive regulator of plant tolerance to salt, osmotic and drought stresses. J. Plant Res. 125, 429-438. doi: 10.1007/s10265011-0448-4

Tsugama, D., Liu, S., and Takano, T. (2012). A bZIP Protein, VIP1, is a regulator of osmosensory signaling in Arabidopsis. Plant Physiol. 159, 144-155. doi: 10. 1104/pp.112.197020

van Leene, J., Blomme, J., Kulkarni, S. R., Cannoot, B., De Winne, N., Eeckhout, D., et al. (2016). Functional characterization of the Arabidopsis transcription factor bZIP29 reveals its role in leaf and root development. J. Exp. Bot. 67, 5825-5840. doi: 10.1093/jxb/erw347

Vision, T. J., Brown, D. G., and Tanksley, S. D. (2000). The origins of genomic duplications in Arabidopsis. Science 290, 2114-2117. doi: 10.1126/science.290. 5499.2114

Wang, J., Zhou, J., Zhang, B., Vanitha, J., Ramachandran, S., and Jiang, S. Y. (2011). Genome-wide expansion and expression divergence of the basic leucine zipper 
transcription factors in higher plants with an emphasis on sorghum. J. Integr. Plant Biol. 53, 212-231. doi: 10.1111/j.1744-7909.2010.01017.x

Wang, Z., Cheng, K., Wan, L., Yan, L., Jiang, H., Liu, S., et al. (2015). Genomewide analysis of the basic leucine zipper (bZIP) transcription factor gene family in six legume genomes. BMC Genomics 16:1053. doi: 10.1186/s12864-0152258-x

Wei, K., Chen, J., Wang, Y., Chen, Y., Chen, S., Lin, Y., et al. (2012). Genomewide analysis of bZIP-encoding genes in maize. DNA Res. 19, 463-476. doi: $10.1093 /$ dnares/dss026

Wigge, P. A., Kim, M. C., Jaeger, K. E., Busch, W., Schmid, M., Lohmann, J. U., et al. (2005). Integration of spatial and temporal information during floral induction in Arabidopsis. Science 309, 1056-1059. doi: 10.1126/science.1114358

Wilkins, M. R., Gasteiger, E., Bairoch, A., Sanchez, J. C., Williams, K. L., Appel, R. D., et al. (1999). Protein identification and analysis tools in the ExPASy server. Methods Mol. Biol 112, 531-552. doi: 10.1385/1-59259-584-7:531

Xu, X., Pan, S., Cheng, S., Zhang, B., Mu, D., Ni, P., et al. (2011). Genome sequence and analysis of the tuber crop potato. Nature 475, 189-195. doi: 10.1038 /nature 10158

Yang, O., Popova, O. V., Suthoff, U., Luking, I., Dietz, K. J., and Golldack, D. (2009). The Arabidopsis basic leucine zipper transcription factor AtbZIP24 regulates complex transcriptional networks involved in abiotic stress resistance. Gene 436, 45-55. doi: 10.1016/j.gene.2009.02.010
Zhang, X., Henriques, R., Lin, S. S., Niu, Q. W., and Chua, N. H. (2006). Agrobacterium-mediated transformation of Arabidopsis thaliana using the floral dip method. Nat. Protoc. 1, 641-646. doi: 10.1038/nprot. 2006.97

Zhou, X. T., Jia, L. J., Wang, H. Y., Zhao, P., Wang, W. Y., Liu, N., et al. (2018). The potato transcription factor StbZIP61 regulates dynamic biosynthesis of salicylic acid in defense against Phytophthora infestans infection. Plant J. 95, 1055-1068. doi: $10.1111 /$ tpj. 14010

Conflict of Interest: XL, DW, and LX were employed by China Tobacco Hunan Industrial Co., Ltd.

The remaining authors declare that the research was conducted in the absence of any commercial or financial relationships that could be construed as a potential conflict of interest.

Copyright $\odot 2021$ Wang, Guo, Li, Sun, Wang, Xu, Li and Guo. This is an open-access article distributed under the terms of the Creative Commons Attribution License (CC BY). The use, distribution or reproduction in other forums is permitted, provided the original author(s) and the copyright owner(s) are credited and that the original publication in this journal is cited, in accordance with accepted academic practice. No use, distribution or reproduction is permitted which does not comply with these terms. 